\title{
On muon bundles from the Universe
}

\author{
P. Kankiewicz ${ }^{1}$, M. Rybczyński ${ }^{1, \star}, Z$. Włodarczyk ${ }^{1}$, and G. Wilk ${ }^{2}$, \\ ${ }^{1}$ Institute of Physics, Jan Kochanowski University, 25-406 Kielce, Poland \\ ${ }^{2}$ National Centre for Nuclear Research, Department of Fundamental Research, 00-681 Warsaw, Poland
}

\begin{abstract}
Recently the CERN ALICE experiment, in its dedicated cosmic ray run, observed muon bundles of very high multiplicities, thereby confirming similar findings from the LEP era at CERN (in the CosmoLEP project). We found significant evidence for anisotropy of arrival directions of the observed high multiplicity muonic bundles. The distribution on celestial sphere and the estimated directionality suggests their possible extragalactic source. We argue that muonic bundles of highest multiplicity are produced by strangelets, hypothetical stable lumps of strange quark matter infiltrating our Universe.
\end{abstract}

\section{Introduction}

This talk is based on [1], where the relevant details can be found. Cosmic ray physics is our unique source of information on events in the energy range which will never be accessible in Earth-bound experiments $[2,3]$. This is why one of the most important aspects of their investigation is the understanding of the primary cosmic ray (CR) flux and its composition. In this respect the recent measurement performed by the ALICE experiment at CERN LHC in its dedicated cosmic ray run $[4,5]$ is of great importance. A number of events with muon bundles of high multiplicity was registered in the so called Extensive Air Showers (EAS) produced by cosmic ray interactions in the upper atmosphere [4]. A special emphasis has been given to the study of high multiplicity events containing more than 100 reconstructed muons (in particular, we were interested in their directionality). Similar events have already been studied in the previous LEP experiments at CERN (in the so-called CosmoLEP program) such as ALEPH [6], DELPHI [7] and L3 [8], and in the underground Baksan experiment [9].

In this talk we shall concentrate mainly on the first, high multiplicity events. To describe them we propose seriously to consider, for a moment, the possibility of the existence in the flux of incoming $\mathrm{CR}$ a component with very high atomic number, of the order of $A \sim 10^{3}$. In fact we propose to return to our old idea that muon bundles of extremely high multiplicities could be produced by strangelets, hypothetical stable lumps of strange quark matter (SQM) infiltrating our Universe [10]. Strangelets with such masses, much larger than the masses of ordinary nuclei, could easily produce extremely large groups of muons in collisions with the atmosphere.

\section{Searches for Strange Quark Matter in cosmic rays}

The idea of SQM originated some time ago in Refs. [11-14], but it remains alive, cf. [15-21]. In short, the basic, commonly accepted view is that that SQM (understood as a combination of roughly an equal

^e-mail: maciej.rybczynski@ujk.edu.pl 


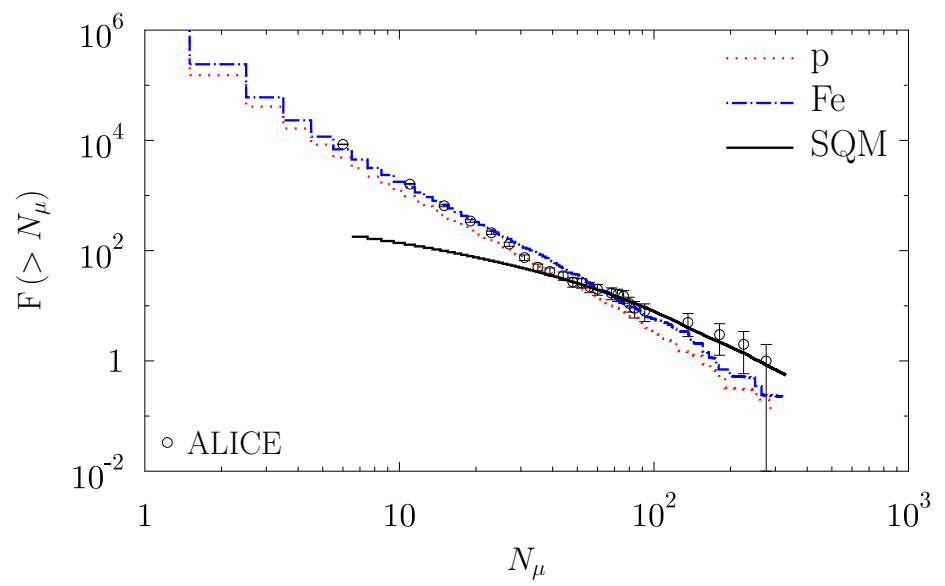

Figure 1. Integral multiplicity distribution of muons for the ALICE data (circles) published in [4]. Monte Carlo simulations for primary protons (dotted line); iron nuclei (dashed dot line) and primary strangelets with mass A taken from the $A^{-7.5}$ distribution (full line) with abundance of the order of $2 \cdot 10^{-5}$ of the total primary flux.

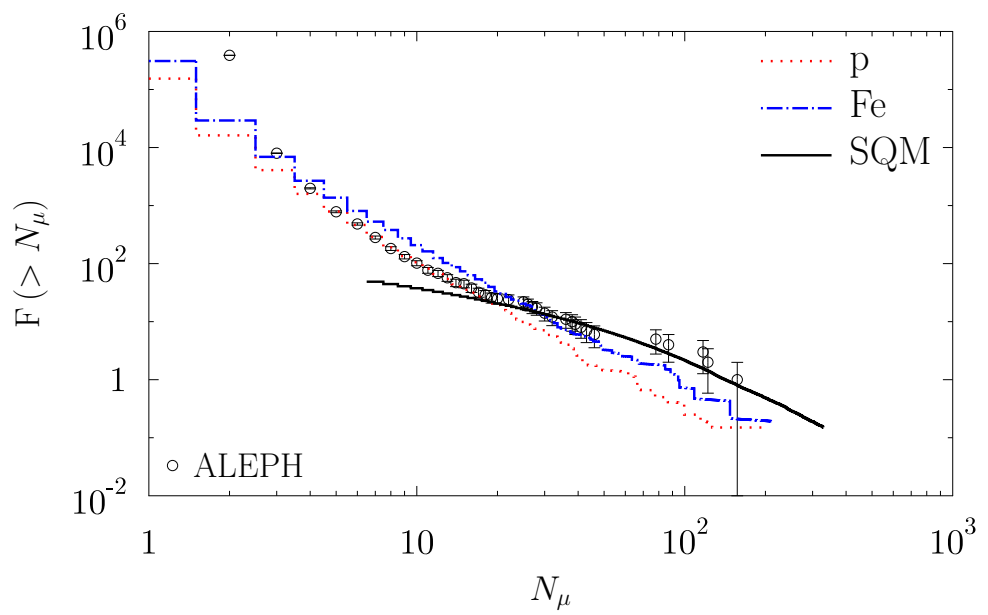

Figure 2. Integral multiplicity distribution of muons the CosmoLEP data (circles) published in [6]. Monte Carlo simulations for primary protons (dotted line); iron nuclei (dashed dot line) and primary strangelets with mass A taken from the $A^{-7.5}$ distribution (full line) with abundance of the order of $2 \cdot 10^{-5}$ of the total primary flux.

number of up, down and strange quarks) might be the true ground state of quantum chromodynamics (QCD). Therefore, it is reasonable to expect that it exists in some form in the Universe and can be detected. This supposition resulted in a number of searches for strange stars and quark stars, in which such a form of matter would be dominant and which could therefore be a possible source of strangelets penetrating outer space [22-29]. The experimental data mentioned above lead to a flux of strangelets which follows the $A^{-7.5}$ behaviour, which in turn coincides with the behaviour of the abundance of 


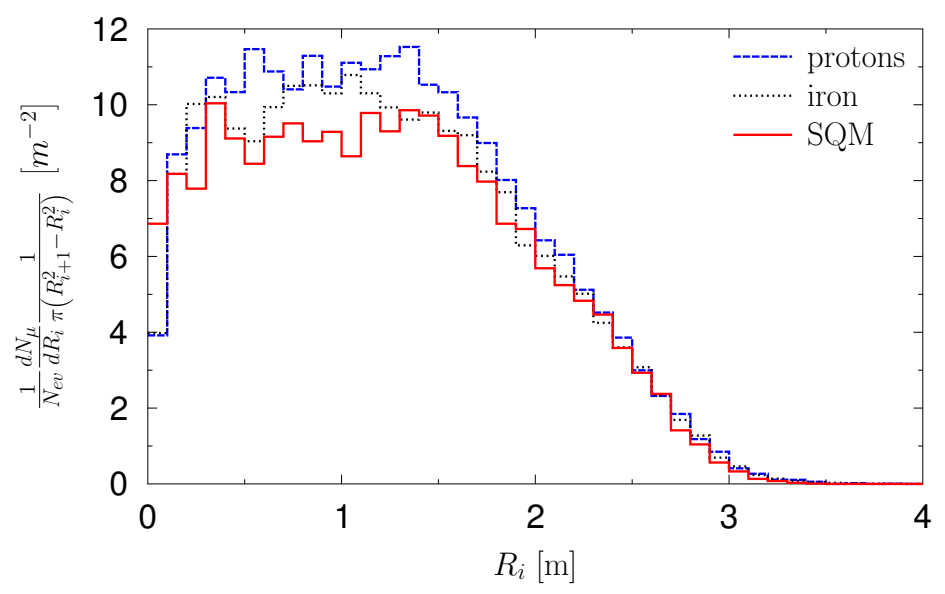

Figure 3. Lateral distribution of muons produces in bundles with $N_{\mu}>100$. Monte Carlo simulations using ALICE experimental setup [4] for primary protons (dashed line); iron nuclei (dotted line) and primary strangelets with mass A taken from the $A^{-7.5}$ distribution (full line).

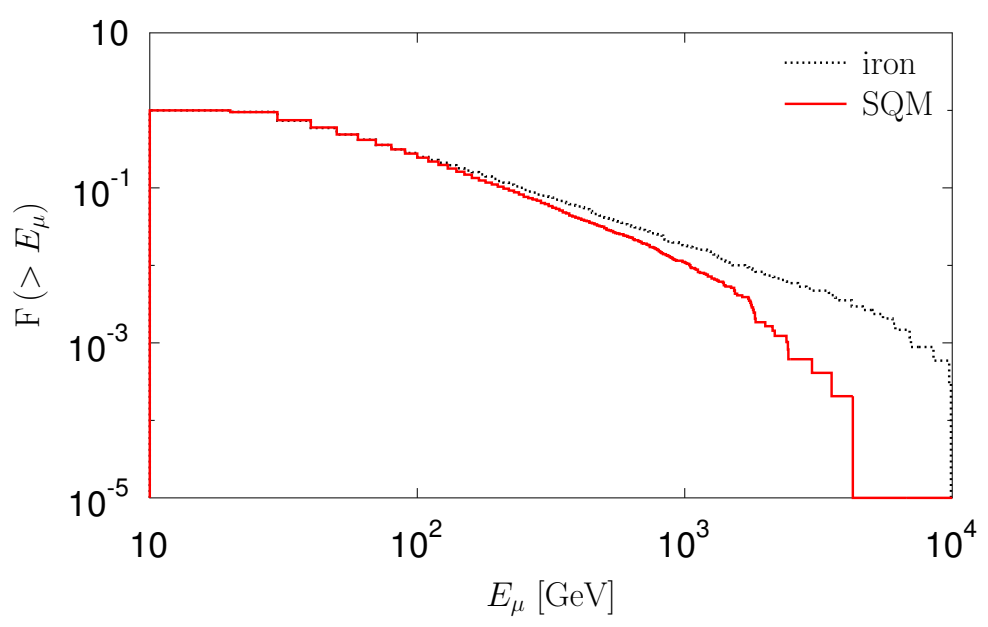

Figure 4. Energy distribution of muons produces in bundles with $N_{\mu}>100$. Monte Carlo simulations using ALICE experimental setup [4] for primary iron nuclei (dotted line) and primary strangelets with mass A taken from the $A^{-7.5}$ distribution (full line).

normal nuclei in the Universe. Strange quark matter fills a vast gap in the distribution of all known forms of stable matter, cf. chart of nuclides presented in Ref. [30]. It places itself exactly between the heaviest atomic elements and neutron stars. The estimated flux of strangelets is also consistent with the astrophysical limits and with the upper limits given experimentally [31]. 


\section{High multiplicity muon bundles from SQM}

We start with a short reminder of our proposed possible scenario of propagation of strangelets through the atmosphere [32, 33]. The apparent contradiction between the large initial size of the incoming strangelets and their required strong penetrability in the atmosphere can be resolved by assuming that strangelets reaching deeply into the atmosphere are formed from the original large strangelet which loses mass in many successive interactions with air nuclei when penetrating the atmosphere. To provide numerical estimate we limit ourselves to the two most extreme pictures of the collision of a strangelet of mass number $A$ with an air nucleus target of mass number $A_{t}$ :

(i) All quarks of $A_{t}$ which are located in the geometrical intersection of the two colliding projectiles are involved and one assumes that each quark from the target interacts with only one quark from the strangelet.

(ii) All quarks from both nuclei which are in their geometrical intersection region participate in the collision.

In the first case, during the interaction up to $\left(3 A_{t}\right)$ quarks from the strangelet could be used up and its mass could drop to a value of $A-A_{t}$, at most. The total penetration depth of the strangelet can be estimated to be in this case equal to

$$
\Lambda \simeq \frac{1}{3} \lambda_{N A_{t}}\left(\frac{A_{0}}{A_{t}}\right)^{\frac{1}{3}}\left(1-\frac{A_{\text {crit }}}{A_{0}}\right)^{2}\left(4-\frac{A_{\text {crit }}}{A_{0}}\right) \simeq \frac{4}{3} \lambda_{N A_{t}}\left(\frac{A_{0}}{A_{t}}\right)^{\frac{1}{3}}
$$

(here $\lambda_{N A_{t}}$ denotes the mean free path for $N-A_{t}$ collisions). One accommodates here both the most probable "normal" mean free paths for successive interactions and the final large penetration depth. This scenario is fully consistent with all present and proposed experiments and could be additionally checked only by measuring the products of the intermediate collisions, which so far is impossible.

The second case is an analogue of the so called tube model used occasionally in nuclear collisions (cf., for example, [34]). After the collision the atomic mass of the strangelet diminishes to a value equal to $A-A^{1 / 3} \cdot A_{t}^{2 / 3}$. While this is a rather extreme variant, it is still useful in providing an estimate of the maximum possible destruction of the quarks in the strangelet.

In this study we have used suitably modified SHOWERSIM [35] modular software. We performed Monte Carlo simulations for primary nuclei composed of protons, iron nuclei, and for primary strangelets with mass $A$ taken from the $A^{-7.5}$ distribution. The results of our simulations are shown in Fig. 1. Integral distributions of muons originating from ordinary nuclei (protons or irons) exhibit power-law dependence, $F\left(>N_{\mu}\right) \sim N_{\mu}^{-2.8}$. Note that whereas the lower and medium multiplicities can be reproduced by the ordinary nuclei, the extremely large groups of muons can be described only by allowing (a relatively minute, of the order of $10^{-5}$ of the total primary flux) admixture of SQM of the same total energy. Fig. 2 shows, for comparison, similar results obtained for the CosmoLEP data [6].

It seems that there are experimental possibilities which allow to distinguish between different types of primaries. Namely lateral, cf. Fig. 3 or energy (Fig. 4) distributions of muons registered in highest multiplicity bundles (here $N_{\mu}>100$ ) may be used for such purpose. Although the lateral distributions of high muon multiplicity groups generated by different types of primary cosmic rays are rather similar (Fig. 3), it is relatively easy to disentangle different primaries by looking at the energy distribution of these muons.

\section{Anisotropy of arrival directions of strangelets}

The ALICE data also turns out to be very valuable for another reason. Namely, they show the angular distribution of the muon events in the spherical reference frame with zenith angle $(\theta)$ and azimuth 
angle $(\Phi)$. This reference system was used in the experiment and is related to the geographical location of the detector.

Taking into account the orientation of the axes, these coordinates can be transformed into a horizontal reference frame: $(\Phi, \theta) \rightarrow(A z, h)$, where $A z$ is the Azimuth used in navigation/astronomy (in this particular case: $A z=\Phi+\alpha_{0}$, where $\alpha_{0}$ is the angle between the $x$ axis of detector reference frame and the direction to the north), and $h$ is the elevation above the horizon $\left(h=90^{\circ}-\theta\right)$. Finally, we can transform the coordinates from the horizontal to the equatorial reference frame: $(A z, h) \rightarrow(\alpha, \delta)$. The equatorial system of coordinates, expressed by right ascension $(\alpha)$ and declination $(\delta)$, related to particular epoch (e.g. 2000.0) is commonly used in astronomy.

Using the usual spherical triangle formulas or rotation matrices one can convert between the horizontal and equatorial coordinates. For the five high-multiplicity muon events we obtained the celestial equatorial coordinates $\left(\alpha_{2000}, \delta_{2000}\right)$ and the estimated limits of their positional errors are $\sim 10^{\circ}$ [1]. The centroid (average location) of the five considered events is marked by a star in Fig. 5. The coordinates of this centroid are: $\alpha_{\text {centr }}=11 \mathrm{~h} 15 \mathrm{~m}, \delta_{\text {centr }}=39^{\circ} 29^{\prime}$. Although these five events seem dispersed near this centroid at a distance of few degrees, they are all located far from the plane of our galaxy. It is worth to notice that the position of the above mentioned centroid is located close to the center of the region registered by the Telescope Array Collaboration data [38]: $\alpha_{T A}=9 \mathrm{~h} 54 \mathrm{~m}$, $\delta_{T A}=44^{\circ} 36^{\prime}$. The angular separation between the centroid and center of the area determined from the TAC data is only $15^{\circ} 48^{\prime}$. Considering the dispersion of five events, possible errors and a very small statistical sample, it is possible to suggest agreement of these results.

We can conclude that the distribution of the directions of these five events suggests their possible extragalactic provenance. Most of them are located in the vicinity of the galactic north pole, far from the galactic plane. If we temporarily assume that all these events have the same origin, the most probable source would be the blazar Mrk 421 which is located close to the centroid of the five events. It is one of the brightest blazars known, with major outbursts, active at all wavebands [36]. However, there are also many other known extragalactic high energy sources located close to the coordinates of our events [36], [37] and, at the moment, they cannot be excluded as potential candidates for the cause of the investigated phenomena.

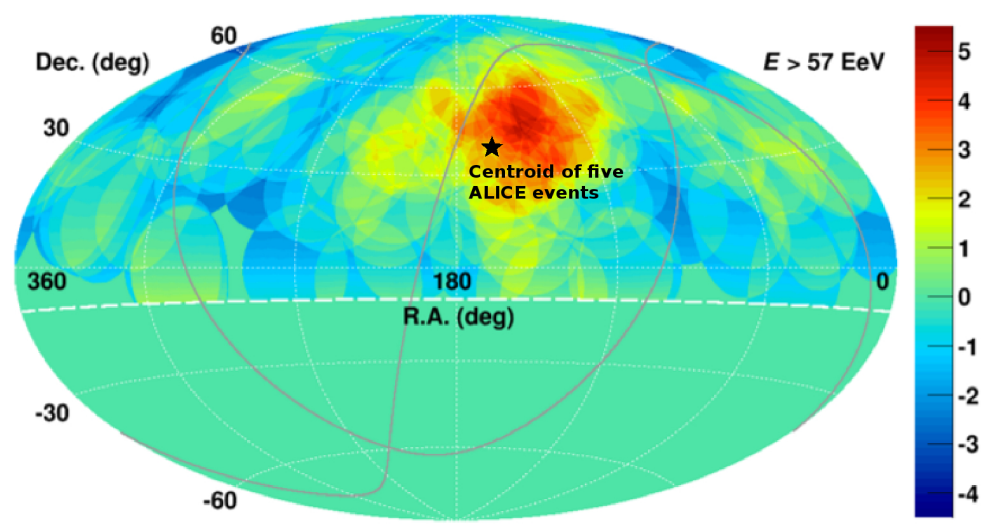

Figure 5. Aitoff projection of the UHECR map in equatorial coordinates taken from Telescope Array Collaboration data [38]. Location of the centroid of five ALICE events is additionally marked with a star. 


\section{Conclusions}

There is an ongoing discussion concerning the possibility of the existence in the Universe of some stable forms of SQM and the feasibility of its detection. Whereas until now we do not have fully convincing observations of SQM, nevertheless there are numerous indications of the possible existence of strange stars, or even of the existence of quark stars. Strangelets could originate, for example, from violent collisions between such objects. The recent results of ALICE reporting the existence of bundles of high-multiplicity muons could serve, notwithstanding the weakness of this signal, as a direct (almost) signal of stable lumps of SQM called strangelets arriving at the Earth from outer space. As a result, we have found that whereas the low multiplicities of muon groups measured by the CERN ALICE experiment favour light nuclei as primaries and the medium multiplicities show behaviour specific for heavier primaries, the muon groups (bundles) of really high multiplicities (of the order of 100) apparently cannot be described by the common interaction models. We have shown here that the situation can be rescued by allowing for a relatively small (of the order of $10^{-5}$ of the total primary flux) admixture of strangelets of the same total energy. Our estimation of their flux does not contradict the results obtained recently by the SLIM Collaboration [31]. The ALICE data allowed us to estimate the directionality of the five events of the highest muon multiplicities, and to identify their most probable extragalactic source(s). The compatibility with other results, especially with the direction of events published by the Telescope Array Collaboration [38] remains very interesting.

\section{Acknowledgments}

This research was supported by the Polish National Science Centre, grant 2016/23/B/ST2/00692 (MR) and contract 2016/22/M/ST2/00176 (GW).

\section{References}

[1] P. Kankiewicz, M. Rybczynski, Z. Włodarczyk and G. Wilk, Astrophys. J. 839, no. 1, 31 (2017).

[2] A. Dar and A. De R ujula, Phys. Rep. 466179 (2008).

[3] A. Letessier-Selvon and T. Stanev, Rev. Mod. Phys. 83907 (2011).

[4] J. Adam et al. [ALICE Collaboration], J. Cosm. Astrop. Phys. 01032 (2016).

[5] J. Adam et al. [ALICE Collaboration], ALICE-PUBLIC-2016-003.

[6] V. Avati et al., Astropart. Phys. 19513 (2003).

[7] J. Abdallah et al. [DELPHI Collaboration], Astropart. Phys. 28273 (2007).

[8] P. Achard et al. [L3 Collaboration], Phys. Lett. B 59815 (2004); [hep-ex/0408114].

[9] V. N. Bakatanov, Yu. F. Novoseltsev and R. V. Novoseltseva, Astrop. Phys. 859 (1997).

[10] M. Rybczyński, Z. Włodarczyk, G. Wilk, Nucl. Phys. B (Proc. Suppl.) 97, 85 (2001).

[11] H. Terazawa, INS-Report - 336 (INS, University of Tokyo, Tokyo) May, 1979.

[12] E. Witten, Phys. Rev. D 30272 (1984).

[13] E. Farhi and R. L. Jaffe, Phys. Rev. D 302379 (1984).

[14] C. Alcock and E. Farhi, Phys. Rev. D 321273 (1985).

[15] J. Madsen, in: J. Cleymans, H. Geyer and F. Scholtz (eds) Lecture notes in physics 516, Springer, Berlin, p. 162.

[16] M. G. Alford, Nucl. Phys. A 830 385c (2009).

[17] F. Weber, Prog. Part. Nucl. Phys., 54193 (2005).

[18] R. Xu, Chin. J. Astron. Astrophys. Suppl. 5353 (2005). 
[19] R. Klingenberg, J. Phys. G 27475 (2001).

[20] H. Terezawa, Nonl. Phen. Compl. Systems 1825 (2015).

[21] H. Terezawa, Nonl. Phen. Compl. Systems 19147 (2016).

[22] C. Alcock, E. Fahri and A. Olinto, Astrop. J. 310261 (1986).

[23] K. S. Cheng, Z. G. Dai and T. Lu, Int. J. Mod. Phys. D 7139 (1998).

[24] K. S. Cheng, Z. G. Dai, D. M. Wei and T. Lu, Science 280407 (1998).

[25] J. J Drake et al., Astroph. J. 572996 (2002).

[26] A. Bauswein, H.-T. Janka, R. Oechslin, G. Pagliara, I. Sagert, J. Schaffner-Bielich, M. M. Hohle and R. Neuhaüser, Phys. Rev. Lett. 103011101 (2009).

[27] E.Finch, J. Phys. G 32 S251 (2006).

[28] W. Kluźniak and W. H. Lee, Mon. Not. R. Astron. Soc. 335 L29 (2002).

[29] Z. Kovács, K. S. Cheng and T. Harko, Astr. Astroph. 500621 (2009).

[30] H. J. Crawford and C. H. Greiner, Sci. Am. 27058 (1994).

[31] Z. Sahnoun et al., Radiat. Meas. 44 894(2009).

[32] G. Wilk and Z. Wlodarczyk, Acta Phys. Hung. 4395 (1996) [hep-ph/9606401].

[33] G. Wilk and Z. Wlodarczyk, J. Phys. G 232057 (1997).

[34] F. Takagi, Prog. Theor. Phys. 65948 (1981).

[35] A. Wrotniak, SHOWERSIM/84, University of Maryland Preprint, 85 (1984).

[36] D. Horan and T. C. Weekes, New Astr. Rev. 48527 (2004).

[37] C. F. Turley et al. [Astrophysical Multimessenger Observatory Network) Collaboration], Astrophys. J. 833, no. 1, 117 (2016).

[38] R. U. Abbasi et al. [Telescope Array Collaboration], Astrophys. J. 790, L21 (2014). 